\title{
BMJ Open Multicentre, open-label, randomised controlled clinical trial to assess the efficacy and safety of appropriate target values for lipid management in patients who have mild-to-moderate stenotic lesions with high-risk plaques in coronary arteries: study protocol
}

\author{
Morihiro Matsuda, ${ }^{1,2}$ Akiko Kada, ${ }^{2}$ Akiko M Saito, ${ }^{2}$ Koji Hasegawa ${ }^{3}$
}

\begin{abstract}
To cite: Matsuda M, Kada A, Saito AM, et al. Multicentre, open-label, randomised controlled clinical trial to assess the efficacy and safety of appropriate target values for lipid management in patients who have mild-to-moderate stenotic lesions with high-risk plaques in coronary arteries: study protocol. BMJ Open 2019;9:e022843. doi:10.1136/ bmjopen-2018-022843

- Prepublication history for this paper is available online. To view these files, please visit the journal online (http://dx.doi. org/10.1136/bmjopen-2018022843).
\end{abstract}

$\mathrm{MM}$ and $\mathrm{AK}$ contributed equally.

Received 14 March 2018 Revised 13 November 2018 Accepted 11 December 2018

Check for updates

(C) Author(s) (or their employer(s)) 2019. Re-use permitted under CC BY-NC. No commercial re-use. See rights and permissions. Published by BMJ.

For numbered affiliations see end of article.

Correspondence to Dr Morihiro Matsuda; mmatsuda.kmc@gmail.com

\section{ABSTRACT}

Introduction To detect patients at high risk of developing myocardial infarction, plaque characteristics as well as the degree of stenosis in coronary arteries should be evaluated. However, unstable plaque or severe calcification detected via coronary artery CT (CACT) is not reflected in risk stratification according to current guidelines. It is hypothesised that patients with high-risk findings on CACT (even those without proven history of coronary artery diseases; CAD) should be strictly managed to lower their low-density lipoprotein cholesterol (LDL-C) levels to targets of secondary prevention. Currently, however, there is no evidence based on prospective randomised intervention studies to prove this hypothesis.

Methods and analysis Patients with mild-to-moderate stenotic lesions with positive remodelling or severe calcification, but without any history of $\mathrm{CAD}$, will be randomly allocated to group A (reduce LDL-C to $<120 \sim 160 \mathrm{mg} / \mathrm{dL}$ according to the primary prevention criteria based on the Japan Atherosclerosis Society (JAS) Guideline for Prevention of Atherosclerotic Cardiovascular Diseases 2017) and group $B$ (reduce $\mathrm{LDL}-\mathrm{C}$ to $<70 \mathrm{mg} / \mathrm{dL}$ according to the secondary prevention criteria for high risk based on the JAS Guideline). They will be strictly managed to achieve the LDL-C targets. We will follow-up and evaluate the composite endpoints consisting of major cardiovascular events (death from CAD, non-fatal myocardial infarction, operation for coronary revascularisation and stroke) and stenosis progression or new stenosis development for 3 years.

Ethics and dissemination The study was approved by the National Hospital Organization Central Research Ethics Committee. The results of this study are scheduled to be published within 2 years after study completion via conference presentation or journal publication. Trial registration number UMIN000031136.

\section{INTRODUCTION}

Effective prevention of myocardial infarction is significantly important for

\section{Strengths and limitations of this study}

- This is the first intervention trial evaluating a prevention strategy in patients with mild-to-moderate stenotic lesions with high-risk characteristics detected on coronary artery computed tomography (CACT), but without proven coronary artery diseases (CAD).

- The primary prevention criteria for reducing low-density lipoprotein cholesterol (LDL-C) to $<120 \sim 160 \mathrm{mg} / \mathrm{dL}$ and secondary prevention criteria for reducing $\mathrm{LDL}-\mathrm{C}$ to $<70 \mathrm{mg} / \mathrm{dL}$ will be compared in this trial, even though most previous trials investigating the effects of LDL-C-lowering therapy have compared dose or strength of statins or other medicines.

- Stenosis progression or new stenosis development detected on CACT, as well as major cardiovascular events, are included as constituent endpoints of primary outcome.

- The study will be conducted in Japan and most enrolled subjects will be Japanese hence, this study cannot conclude on the effects of prevention strategy in general populations.

- The study design involves an open-label trial because without knowing the allocated group, participants and care-providers will not be able to achieve the assigned LDL-C target during the trial.

prolonging healthy life and suppressing surging medical costs. Myocardial infarction is not necessarily caused by a severe stenotic lesion and is known to be often caused by the rupture of an unstable plaque in a mildto-moderate stenotic lesion. ${ }^{1}$ This implies that, in order to find patients at a high risk of developing myocardial infarction, plaque 
characteristics, rather than the degree of stenosis of the coronary artery, should be evaluated.

However, cardiac catheter examination (coronary angiography; CAG), which is considered the gold standard for diagnosis of coronary artery disease (CAD), excels in the evaluation of the degree of stenosis but cannot evaluate plaque characteristics. Therefore, it is necessary to add invasive examinations including intravascular endoscopy, intravascular ultrasound (IVUS) or optical coherence tomography for the evaluation.

Coronary artery CT (CACT) is a non-invasive examination that can be performed on an outpatient basis. It is conducted in more than 400000 patients annually and its usage is increasing year by year in Japan. ${ }^{2}$ Although CACT is inferior to cardiac catheterisation at evaluating stenosis accurately, it is excellent in the evaluation of plaque characteristics on the blood-vessel wall. It has been reported that findings such as a low attenuation plaque, positive remodelling and a napkin-ring sign suggest the presence of unstable plaques ${ }^{34}$ and the presence of these unstable plaques is associated with a high prevalence of acute coronary syndrome (ACS) ${ }^{5}$ In addition, coronary artery calcification score (CACS) obtained from CACT is useful for prognosis; in cases with high values of CACS, it has been reported that death from cardiovascular events, including myocardial infarction, occurs at a high rate. ${ }^{6}$

The Japan Atherosclerosis Society (JAS) Guidelines for Prevention of Atherosclerotic Cardiovascular Diseases $2017^{7}$ recommend lipid management with target low-density lipoprotein cholesterol (LDL-C) levels of less than $100 \mathrm{mg} / \mathrm{dL}$ as secondary prevention when there is coronary artery stenosis or a history of myocardial infarction, and strict management targeting LDL-C levels of less than $70 \mathrm{mg} / \mathrm{dL}$ is required for pathologies which are at high risk of developing atherosclerotic diseases including ACS. Under similar guidelines in Europe and the USA, ${ }^{89} \mathrm{CAD}$ is classified to be at very high risk and lipid management with target LDL-C levels of less than $70 \mathrm{mg} / \mathrm{dL}$ is recommended. However, when patients do not have proven $\mathrm{CAD}$, the presence of unstable plaque or hyper-calcification detected in CACT is not reflected in risk stratification. Therefore, patients with high-risk plaques but without ACS onset are only required to achieve the LDL-C goals for primary prevention according to the current guidelines. Several clinical trials using IVUS have revealed that lowering- LDL-C levels to less than $70 \mathrm{mg} / \mathrm{dL}$ was effective for regression of coronary artery plaque volume. ${ }^{10-12}$ In particular, the ESTABLISH study demonstrated that strict LDL-C -lowering to $70 \mathrm{mg} / \mathrm{dL}$ resulted in a $13.1 \%$ reduction in plaque volume in 6 months, ${ }^{10}$ which might have led to a significant reduction in cardiovascular events in a follow-up for 4.2 years. ${ }^{11}$ In PRECISE-IVUS study, a combination of statins and ezetimibe, which lowered average LDL-C levels to $70 \mathrm{mg} / \mathrm{dL}$, reduced plaque volume more effectively than statins alone. ${ }^{12}$ Therefore, in the current study in patients with high-risk plaques detected on CACT but without proven history of CAD, we will investigate if strict management targeting LDL-C levels of less than $70 \mathrm{mg} / \mathrm{dL}$ can prevent cardiovascular events and progression of coronary artery plaques more effectively than standard management of LDL-C for primary prevention.

\section{METHODS/DESIGN \\ Study design}

This is a multicentre, open-label, randomised, controlled, parallel-group clinical study. The complete design of this trial is shown in figure 1.

The following 16 institutions will participate in this study in which the eligibility and outcomes will be judged by experienced specialists for interpretation of CACT: Hokkaido Medical Center, Hakodate National Hospital, Mito Medical Center, Saitama National Hospital, Yokohama Medical Center, Sagamihara National Hospital, Kanazawa Medical Center, Kyoto Medical Center, Osaka National Hospital, Okayama Medical Center, Kure Medical Center, Iwakuni Clinical Center, Kobe Medical Center, Ehime Medical Center, Kyushu Medical Center and Kumamoto Medical Center.

\section{Objective}

Primary endpoint

To investigate the efficacy of strict lipid management by secondary prevention high-risk criteria for preventing major cardiovascular events and progression of coronary artery stenosis in study subjects without proven history of CAD who have mild to moderate stenosis with unstable plaques or severe calcification detected by CACT, in comparison with standard lipid management as per the primary prevention criteria.

\section{Major secondary endpoints}

To evaluate the safety and efficacy in preventing each component of primary endpoints by strict lipid management with secondary prevention high-risk criteria in study subjects without proven history of CAD who have mild-tomoderate stenosis with unstable plaques or severe calcification detected by CACT, in comparison with standard lipid management as per primary prevention criteria.

After randomization of the participants into group A or $\mathrm{B}$, they will be treated in an unblinded fashion using any LDL-C lowering therapies to achieve the following LDL-C goals assigned to their group for 3 years.In group A, target values for LDL-C would be 100 <120 mg/dL for high-risk category, $120 \sim<140 \mathrm{mg} / \mathrm{dL}$ for medium-risk category and $140 \sim 160 \mathrm{mg} / \mathrm{dL}$ for low-risk category, according to the primary prevention criteria based on JAS Guidelines. ${ }^{7}$ The three risk categories (low, medium and high) will be determined by the following risk stratification.

\section{Risk stratification}

Any complications of diabetes mellitus, chronic kidney diseases, non-cardiogenic cerebral infarction and peripheral arterial diseases will be classified as high risk. In the 


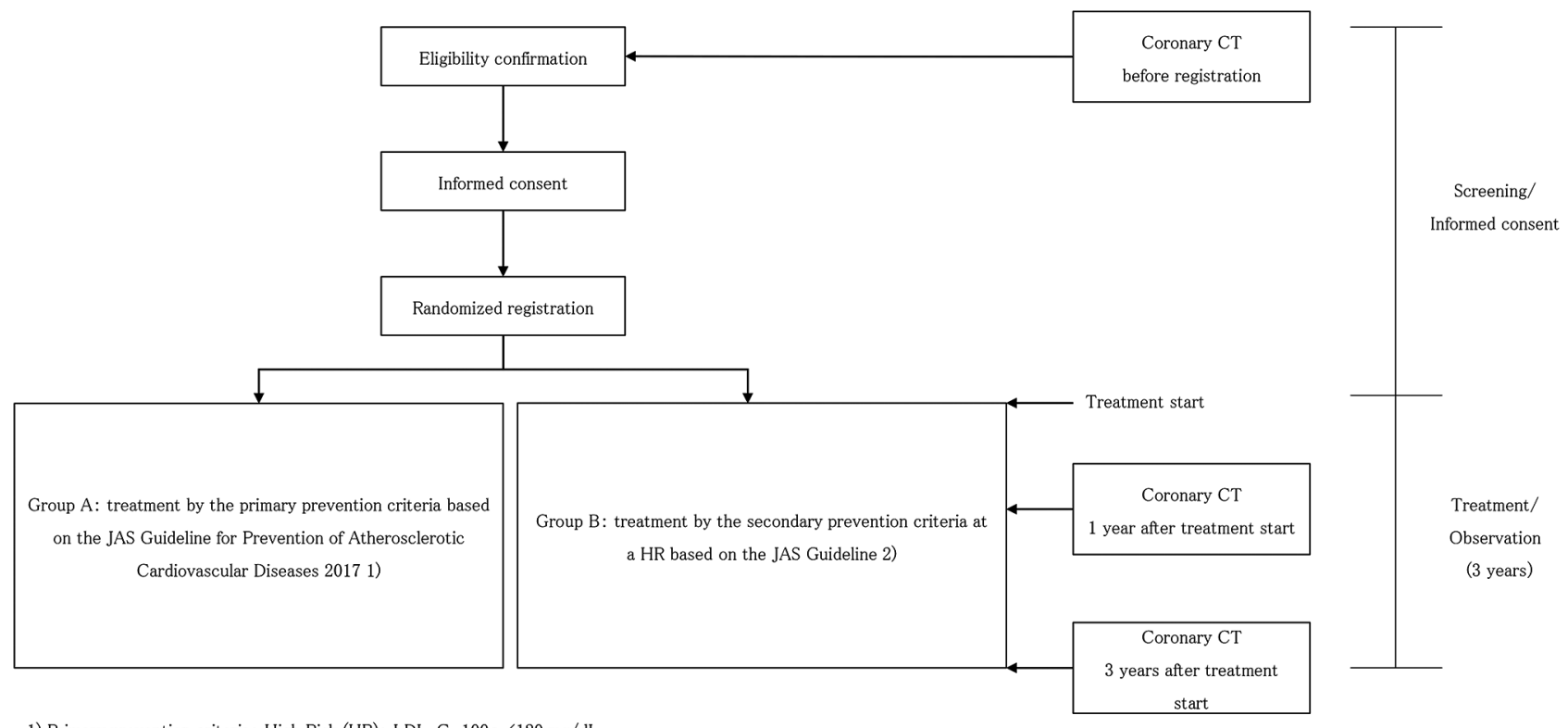

1) Primary prevention criteria: High Risk (HR): LDL-C $100 \sim<120 \mathrm{mg} / \mathrm{dL}$

Intermediate Risk (IR): LDL-C $120 \sim<140 \mathrm{mg} / \mathrm{dL}$

Low Risk (LR): LDL-C $140 \sim<160 \mathrm{mg} / \mathrm{dL}$

2) Secondary prevention HR criteria: $L D L-C:<70 \mathrm{mg} / \mathrm{dL}$

Figure 1 Overall schedule of this study (screening and randomisation to group A and group B, intervention and observation).

absence of these complications, risk stratification would be conducted based on the predictive model of CAD according to the Suita score,$^{13}$ which can be calculated from the following factors: sex, age, smoking/non-smoking status, blood pressure, LDL-C levels, high-density lipoprotein cholesterol levels, impaired glucose tolerance and the presence or absence of a family history of premature CAD. The probability of the onset of CAD will be calculated from the Suita score and categorized as a low risk if less than $2 \%$ for 10 years, a medium risk if $2 \%$ to $9 \%$, and a high risk if $9 \%$ or more.

In group $B$, target value for LDL-C is $<70 \mathrm{mg} / \mathrm{dL}$ according to the secondary prevention criteria for high risk based on the JAS Guidelines. ${ }^{7}$

In the LDL-C-lowering treatment, patients will be guided to diet and exercise therapies to improve lifestyle at first and then, can be additionally treated with the following therapeutic agents, if achieving the assigned target value is considered difficult after 3 months. The LDL-C levels should achieve the target value within 6 months after the start of treatment.

1. Hydroxymethylglutaryl-CoA reductase inhibitor (statin): an inhibitor of cholesterol synthesis in the liver.

2. Small intestinal cholesterol transporter inhibitor (ezetimibe): an inhibitor of cholesterol absorption in the small intestine.

3. Anion exchange resin: a promoter of bile acid excretion.

\section{Sample size}

According to the prospective observational study in patients who underwent CACT, ${ }^{3}$ of 70 patients with vulnerable plaques, seven developed ACS and two developed stable angina, while stenotic progression was observed in
15 patients at the second CT. ACS is composed of acute myocardial infarction and unstable angina applicable to the subject of coronary artery revascularisation in major cardiovascular events included in the primary endpoint of this study or non-fatal myocardial infarction. The events corresponding to the composite outcome of the primary endpoint items of this study occurred in 24 patients (major cardiovascular events: nine patients, stenosis progression: 15 patients), until the second CT was performed to detect stenotic progression (median interval: 366 days, IQR: 203-742 days) and the incidence rate at 3 years was calculated as $34.3 \%(24 / 70)$. According to the meta-analysis of randomised controlled trials using statins, ${ }^{14}$ the comparison of more versus less statin showed that major cardiovascular events were reduced by $228 \%$ when LDL-C was lowered by $1 \mathrm{mmol} / \mathrm{L}(38.7 \mathrm{mg} / \mathrm{dL})$.

Since the subjects are suspected of CAD and have undergone CACT, majority of them are expected to be high-risk patients. Therefore, if the LDL-C values would be managed according to the target values of this study, the estimates in groups A and B would be 100 to $120 \mathrm{mg}$ / $\mathrm{dL}$ and 60 to $70 \mathrm{mg} / \mathrm{dL}$, respectively. When trying to lower LDL-C with strict treatment such as in group B, it is expected that the LDL-C target value will not be achieved. In anticipation of this, the difference between LDL-Cs in groups $\mathrm{A}$ and $\mathrm{B}$ is considered to be about $1 \mathrm{mmol} / \mathrm{L}$ (about $38.7 \mathrm{mg} / \mathrm{dL}$ ). Assuming that the occurrence of the event decreases by about $28 \%$, similar to the results of previous studies, ${ }^{14}$ the incidence of events in 3 years is calculated to be about $34.3 \%$ and $24.7 \%$ in groups A and $B$, respectively. At a significance level of $\alpha=0.025$ (one-sided) and detection power of 0.8 , with 353 patients per group, we will be able to reject the null hypothesis: 
incidence of event in group A is less than group B. Estimating dropouts during the follow-up period to be about $5 \%$, sample size was set as 370 in each group (740 patients in total).

\section{Eligibility criteria}

Inclusion criteria

1. Patients with a mild-to-moderate stenotic lesion in trunk, branches or periphery with a diameter of $2 \mathrm{~mm}$ or more, which has positive remodelling (remodelling index $(\mathrm{RI}) \geq 1.1^{3-5}$ ), or with an advanced coronary artery calcification CACS $>400$ ) detected on CACT.

2. Patients aged 20 years or more at the time of consent.

3. Patients willing to provide written informed consent.

\section{Exclusion criteria}

1. Patients with a significant stenosis of the coronary artery, indicating revascularisation.

2. Patients having a history of myocardial infarction.

3. Patients having a history of coronary artery revascularisation (percutaneous coronary intervention and coronary artery bypass graft).

4. Patients whose LDL-C value already fulfils the secondary prevention criteria in JAS Guidelines for Prevention of Atherosclerotic Cardiovascular Diseases 2017 at the time of registration.

5. Patients diagnosed to have familial hypercholesterolaemia (homozygote).

6. Patients with poorly controlled diabetes, with HbAlc of over $10 \%$.

7. Subjects whose body mass index is less than $17 \mathrm{~kg} / \mathrm{m}^{2}$.

8. Patients with chronic kidney disease, with estimated glomerular filtration rate of $30 \mathrm{~mL} / \mathrm{min} / 1.73 \mathrm{~m}^{2}$ or less.

9. Patients having a history of complications suspected to be related with the contrast medium.

10. Patients with thyroid disease requiring medical treatment.

11. Patients with bronchial asthma, multiple myeloma, macroglobulinaemia, tetany or pheochromocytoma.

12. Patients having a history of side effects caused by statins.

13. Patients in poor compliance with medications due to dementia.

14. Subjects judged to be inappropriate for participation in this study by the principal investigator (PI) and sub-investigators.

\section{Blinding and randomisation}

This will be an open-label, randomised controlled clinical trial. Participants and care-providers will be unblinded to the allocated group, because they cannot achieve the assigned LDL-C target during the trial without its knowledge. However, in judgement of coronary artery stenosis, the Event Evaluation Committee will perform central review with the allocation group blinded, and the central review result would be adopted in the final analysis.
Allocation of the study subjects to each group is conducted by the electronic data capture system using the minimisation method accounting for the following allocation adjustment factors: age (younger than 74 years, or 75 years or older), sex (male or female), LDL-C value before treatment (less than 140, or 140 or more), with or without any other comorbidities [diabetes, chronic kidney disease, non-cardiogenic cerebral infarction and peripheral arterial disease].

\section{Outcomes}

\section{Primary outcomes}

Composite endpoint: major cardiovascular events (death from CAD, non-fatal myocardial infarction, operation for coronary revascularisation, stroke) and stenotic progression or new stenosis development.

\section{Secondary outcomes}

1. All deaths (deaths from heart disease, non-cardiac disease, unexplained cause).

2. ACS onset (non-fatal myocardial infarction, unstable angina).

3. Operation for coronary artery revascularisation.

4. Stenotic progression or new stenosis development.

5. Exacerbation of calcification.

6. Stroke (atherothrombotic cerebral infarction, cardiogenic cerebral embolism, cerebral haemorrhage).

7. Adverse events.

8. Proportion of achievement of target value for LDL-C management.

\section{Assessments}

CT measurements

CACT images can be obtained using 64 or more slice CT according to the protocol optimised in each hospital enrolled in this study. The findings on CACT will be evaluated by two cardiologists of Central Evaluation Committee blinded to the patient's information.

\section{Definition of coronary artery stenosis}

On CACT conducted at baseline, year 1, and year 3, coronary artery stenosis is estimated and graded as none (no luminal stenosis), mild ( $1 \%$ to $39 \%$ ), moderate $(40 \%$ to $69 \%$ ), severe ( $70 \%$ to $99 \%$ ) stenosis or occluded, according to the guidelines of Society of Cardiovascular CT. ${ }^{15}$ Stenotic progression is defined when the degree of stenosis increases by grade 1 or more. New stenosis development is defined when new stenosis higher than mild degree appears. When the stenosis is unevaluable on CACT, the lesion should be re-evaluated by CAG on catheter examination and the stenosis degree on the CAG is adopted for the analysis.

\section{Remodelling index}

Manual inspection, in both cross section and longitudinal reconstruction, is used for defining the coronary artery $\mathrm{RI}$ (RI=lesion diameter/reference diameter) ${ }^{3-5}$ 
Coronary artery calcification score

Calcified lesions with a peak attenuation of more than 130 Hounsfield units are quantified in whole coronary artery wall, and are then scored as CACS using Agatston method. ${ }^{16}$

\section{Assessments of major cardiovascular events}

The diagnosis of myocardial infarction is based on combination of symptoms and ECG findings and elevation of circulating cardiac biomarkers. ${ }^{17}$ The patients with symptoms based on Braunwald classification, but without electrographic changes or elevation in circulating cardiac biomarkers are diagnosed with unstable angina. ${ }^{18}$ Coronary revascularisation would be performed only in patients proven to have coronary stenosis causing ischaemia with any other modalities. Judgement of the events will be conducted by the Central Evaluation Committee.

\section{Serious adverse events report}

We will collect data on all serious adverse events (SAE) from the start to the end of the study treatment or to the 30th day from the discontinuation of study treatment. Following the initial SAE report, the PI or sub-investigators will track these cases until the events are confirmed (death, recovery, lost to follow-up).

\section{Statistical analysis}

In all efficacy evaluations, we establish the Full Analysis Set (FAS) as the main analysis set and Per Protocol Set (PPS) conforming to the study plan as reference. FAS are defined as the data of registered patients who have no serious protocol violations. PPS is defined as the population excluding patients in which there was a violation of eligibility/exclusion criteria or those who refused concomitant medicine or therapy from the FAS. Among all registered patients, a group excluding patients in which no study treatment is performed at all is called the safety analysis population (SAF).

\section{Efficacy analysis}

The superiority test (significance level $\alpha=0.025$ (one-sided)) in the primary endpoint will be carried out using logistic regression analysis with covariates as allocation adjustment factors. For the secondary endpoints, we will examine the relationship with lipid management criteria using the Cox proportional hazard model for all deaths, ACS onset and operation for coronary artery revascularisation, and will explore risk factors related to development of coronary artery plaque and exacerbation of calcification using multivariate analysis with various related factors as explanatory variables.

\section{Safety analysis}

All safety analyses will be carried out for SAF. Frequency of adverse events will be calculated in each group.

\section{Interim analysis}

Existing work on 'progression of the degree of stenosis or emergence of new lesion by CACT' included in the primary endpoint is reported in only one study by Motoyama et $a \check{l}$ and information on the incidence of events is not enough. Therefore, an interim analysis will be performed at the time when the results of follow-up evaluation by CACT at 1 year in 200 registered patients (by judgement at the implementation facility) will be collected and the sample size will be re-estimated. Specifically, using the method of Mehta and Pocock ${ }^{19}$ if the conditional detection power is between $50 \%$ to $80 \%$ at the time when interim analysis data are obtained, the sample size will be re-estimated. The upper limit of the sample size is 1500 .

\section{Data management}

In this study, we will collect data using electronic data capture (EDC). The PI or a person nominated by the PI shall promptly input collected case information to the EDC. The transmitted electronic data would be regarded as a case report. PI will confirm that all data inputs are accurate. The data managers of respective data centres will occasionally monitor for suspicious inputs by the EDC using the central monitoring method.

\section{Independent data monitoring committee}

We will establish an independent data monitoring committee consisting of three experts in cardiology internal medicine and one expert in biostatistics. In response to consultation from the research representative, the committee will assess the efficacy and safety and make recommendations. Moreover, based on the results of interim analysis, we will decide whether to continue the study and validate the study plan modification.

\section{Patient and public involvement}

Patients and public were not involved in designing this study.

\section{DISCUSSION}

In six clinical studies, statins were administered in patients under secondary prevention and the incidence rate of coronary events in the control group (those under treatment without statin administration or standard treatment) was $5.2 \%-9.0 \%$ per year. ${ }^{14}$ Accordingly, coronary artery events within 3 years are presumed to be $15.6 \%$ or more. Meanwhile, according to the observational study conducted by Motoyama et al, 3158 patients who underwent CACT were followed up for an average 3.9 years. As a result, 40 patients $(1.4 \%)$ among a total of 2864 without high-risk plaques developed ACS, whereas 48 patients (16.3\%) among a total of 294 with high-risk plaques developed ACS. ${ }^{5}$ In similar studies conducted by several other groups, it was reported that patients with high-risk plaque detected in CACT have higher incidence rates of coronary events. This means that patients with high-risk plaques detected in CACT are considered to be at risk for coronary artery events similar to patients requiring the secondary prevention. 
In patients with enlarged plaques and stenotic progression confirmed in CACT after 1 year, ACS developed in about $26.7 \%$ cases in the median follow-up period of 4 years. ${ }^{5}$ This applies to a HR of 33.43 (95\% CI of 4.13 to $78.03, \mathrm{p}<0.0001)$ in multivariate analysis, ${ }^{5}$ and enlarged plaques/stenosis development have proven to be strong predictors closely related to the onset of coronary events. Therefore, we included stenosis progression or new stenosis development as a constituent endpoint in our primary outcome.

In the mild calcification group with CACS of 0 to 99 quantified using CACT, death due to the onset of CAD or the onset of myocardial infarction was $0.4 \%$ per year, whereas in the group with high calcification represented by CACS 400 or more, the event risk was significantly high $\left(2.4 \%\right.$ per year) ${ }^{6}$ In addition, when coronary artery calcification increases to CACS more than 100 per year, CAD have been shown to occur at a high rate of $10 \%$ to $15 \%$ in 3 years. ${ }^{16}$ Thus, patients with an unstable plaque or a severe calcification detected in CACT are at high risk of coronary artery events, even if CAD has not yet developed. However, currently there is no evidence based on prospective randomised interventional studies to prove that these high-risk patients should be strictly managed to achieve lower LDL-C goals with a secondary prevention strategy even without no proven CAD.

Treatment criteria in this study are based on recommendations of the Guidelines for Prevention of Atherosclerotic Diseases established based on evidence showing the efficacy and safety in many clinical trials. All drugs and doses to be used in this study are approved and covered by national health insurance in Japan, and it is expected that adverse events in this study will not exceed those occurring in daily clinical practice.

There are several limitations in the current study. This study will be conducted in Japan and most enrolled participants will be Japanese hence, the conclusions cannot be generalised to people of other races, lifestyles or economic statuses. As this study is an open-label trial, confounding factors dependent on the allocated group, other than strict lipid management, may affect the outcomes.

In the SCOT-HEART trial, the use of CACT in addition to standard care in patients with stable chest pain resulted in a significant reduction in major cardiovascular events compared with standard care alone, suggesting that it may be attributed to more prevention therapies initiated in patients undergoing CACT. ${ }^{20}$ However, it is unclear whether strict lipid management improved the outcomes. Our current study will contribute to the development of a better preventive therapy, by clarifying appropriate lipid management criteria for patients who have high-risk coronary atherosclerosis detected on CACT.

\section{Ethics and dissemination}

Prior to this study, the PI or sub-investigators obtained written informed consent from the patients or patients' legally acceptable representatives.
Subject information will be collected using the EDC system. In order to protect personal information, the name, address and telephone number, of the subjects will not be obtained. The medical information will be identified using the case registration number issued.

The results of this study are scheduled to be published within 2 years after study completion, via conference presentation or journal publication.

\section{Author affiliations}

${ }^{1}$ Institute for Clinical Research, National Hospital Organization Kure Medical Center, Kure, Japan

${ }^{2}$ Clinical Research Center, National Hospital Organization Nagoya Medical Center, Nagoya, Japan

${ }^{3}$ Division of Translational Research, National Hospital Organization Kyoto Medical Center, Kyoto, Japan

Acknowledgements The authors are grateful to the NHO Cardiology group.

Contributors MM finally approved the protocol and manages the overall study. $\mathrm{MM}$ and $\mathrm{KH}$ conceived of, planned and will manage the study. AK is responsible for statistical analysis in this study. AMS will perform data management and central monitoring in this study. AK will have access to the final trial dataset, but such access will be limited to other investigators by contractual agreement. MM and AK created and revised the manuscript and all authors read and approved the final manuscript.

Funding The authors have not declared a specific grant for this research from any funding agency in the public, commercial or not-for-profit sectors.

\section{Competing interests None declared.}

Patient consent for publication Not required.

Ethics approval The study was approved by the National Hospital Organization Central Research Ethics Committee on 5 December 2017 (protocol version 1).

Provenance and peer review Not commissioned; externally peer reviewed.

Open access This is an open access article distributed in accordance with the Creative Commons Attribution Non Commercial (CC BY-NC 4.0) license, which permits others to distribute, remix, adapt, build upon this work non-commercially, and license their derivative works on different terms, provided the original work is properly cited, appropriate credit is given, any changes made indicated, and the use is non-commercial. See: http://creativecommons.org/licenses/by-nc/4.0/.

\section{REFERENCES}

1. Casscells W, Hathorn B, David M, et al. Thermal detection of cellular infiltrates in living atherosclerotic plaques: possible implications for plaque rupture and thrombosis. Lancet 1996;347:1447-9.

2. The Japanese Circulation Society. The japanese registry of all cardiac and vascular diseases (jroad); annual report. 2015. In Japanese http://www.j-circ.or.jp/jittai_chosa/jittai_chosa2015web.pdf (Accessed 5 Feb 2018).

3. Motoyama S, Sarai M, Harigaya $\mathrm{H}$, et al. Computed tomographic angiography characteristics of atherosclerotic plaques subsequently resulting in acute coronary syndrome. J Am Coll Cardiol 2009;54:49-57

4. Otsuka K, Fukuda S, Tanaka A, et al. Napkin-ring sign on coronary CT angiography for the prediction of acute coronary syndrome. JACC Cardiovasc Imaging 2013;6:448-57.

5. Motoyama S, Ito $\mathrm{H}$, Sarai $\mathrm{M}$, et al. Plaque characterization by coronary computed tomography angiography and the likelihood of acute coronary events in mid-term follow-up. J Am Coll Cardiol 2015;66:337-46.

6. Greenland P, Bonow RO, Brundage BH, et al. ACCF/AHA 2007 clinical expert consensus document on coronary artery calcium scoring by computed tomography in global cardiovascular risk assessment and in evaluation of patients with chest pain: a report of the American college of cardiology foundation clinical expert consensus task force (accf/aha writing committee to update the 2000 expert consensus document on electron beam computed tomography) developed in collaboration with the society of atherosclerosis imaging and prevention and the society 
of cardiovascular computed tomography. J Am Coll Cardiol 2007:49:378-402.

7. Japan Atherosclerosis Society. JAS Guidelines for Prevention of Atherosclerotic Cardiovascular Diseases, 2017.

8. Catapano AL, Graham I, De Backer G, et al. 2016 ESC/EAS Guidelines for the management of dyslipidaemias. Eur Heart $J$ 2016;37:2999-3058.

9. Jellinger PS, Handelsman Y, Rosenblit PD, et al. American association of clinical endocrinologists and american college of endocrinology guidelines for management of dyslipidemia and prevention of cardiovascular disease. Endocr Pract 2017;23:1-87.

10. Okazaki S, Yokoyama T, Miyauchi K, et al. Early statin treatment in patients with acute coronary syndrome: demonstration of the beneficial effect on atherosclerotic lesions by serial volumetric intravascular ultrasound analysis during half a year after coronary event: the ESTABLISH Study. Circulation 2004;110:1061-8.

11. Dohi T, Miyauchi K, Okazaki S, et al. Early intensive statin treatment for six months improves long-term clinical outcomes in patients with acute coronary syndrome (Extended-ESTABLISH trial): a follow-up study. Atherosclerosis 2010;210:497-502.

12. Tsujita $\mathrm{K}$, Sugiyama S, Sumida $\mathrm{H}$, et al. Impact of dual lipid-lowering strategy with ezetimibe and atorvastatin on coronary plaque regression in patients with percutaneous coronary intervention: the multicenter randomized controlled PRECISE-IVUS Trial. J Am Coll Cardiol 2015;66:495-507.
13. Nishimura K, Okamura T, Watanabe M, et al. Predicting coronary heart disease using risk factor categories for a Japanese urban population, and comparison with the framingham risk score: the suita study. J Atheroscler Thromb 2014;21:784-98.

14. Baigent C, Blackwell L, Emberson J, et al. Efficacy and safety of more intensive lowering of LDL cholesterol: a meta-analysis of data from 170,000 participants in 26 randomised trials. Lancet 2010;376:1670-81.

15. Raff GL, Abidov A, Achenbach S, et al. SCCT guidelines for the interpretation and reporting of coronary computed tomographic angiography. J Cardiovasc Comput Tomogr 2009;3:122-36.

16. Budoff MJ, Young R, Lopez VA, et al. Progression of coronary calcium and incident coronary heart disease events: MESA (Multi-Ethnic Study of Atherosclerosis). J Am Coll Cardiol 2013;61:1231-9.

17. Thygesen K, Alpert JS, Jaffe AS, et al. Third universal definition of myocardial infarction. Circulation 2012;126:2020-35.

18. Braunwald E. Unstable angina. A classification. Circulation $1989 ; 80: 410-4$

19. Mehta CR, Pocock SJ. Adaptive increase in sample size when interim results are promising: a practical guide with examples. Stat Med 2011;30:3267-84.

20. Newby DE, Adamson PD, Berry C, et al. Coronary ct angiography and 5-year risk of myocardial infarction. $N$ Engl $J$ Med 2018;379:924-33. 